\title{
Argumentation and qualitative decision making
}

\author{
Simon Parsons and Shaw Green \\ Department of Electronic Engineering, \\ Queen Mary \& Westfield College, \\ University of London, \\ London E1 4NS, UK \\ \{s.d.parsons, s.d.green\}@elec.qmw.ac.uk
}

\begin{abstract}
This paper presents a system of argumentation which captures the kind of reasoning possible in qualitative probabilistic networks, including reasoning about expected utilities of actions and the propagation of synergies between actions. In these latter regards it is an extension of our previous work on systems of argumentation which reason with qualitative probabilities.
\end{abstract}

\section{Introduction}

In the last few years there have been a number of attempts to build systems for reasoning under uncertainty that are of a qualitative nature - that is they use qualitative rather than numerical values, dealing with concepts such as increases in belief and the relative magnitude of values. Three main classes of system can be distinguished - systems of abstraction, infinitesimal systems, and systems of argumentation. In systems of abstraction, the focus is mainly on modelling how the probabilities of hypotheses change when evidence is obtained. Such systems provide an abstract version of probability theory, known as qualitative probabilistic networks (QPNs) [25], which is sufficient for planning [25], explanation [5] and prediction [18] tasks. Infinitesimal systems deal with beliefs that are very nearly 1 or 0 , providing formalisms that handle order of magnitude probabilities. Such systems may be used for diagnosis [4] and have been extended with infinitesimal utilities to give complete decision theories [21,26]. Systems of argumentation are based on the idea of constructing logical arguments for and against formulae. Such systems of have been applied to problems such as diagnosis, protocol management and risk assessment [11], as well as handling inconsistent information [1], and providing a framework for default reasoning $[10,16]$.

In a previous paper [17], we provided a hybridisation of the argumentation and abstraction approaches by introducing a system called the qualitative probabilistic reasoner $(\mathcal{Q P} \mathcal{R})$ which constructed arguments about how probabilities change. In this paper we extend the kind of reasoning possible using $\mathcal{Q P} \mathcal{R}$ to deal with information about changes in utilities, thus providing a qualitative utility reasoner $\mathcal{Q U} \mathcal{R}$ which provides an abstraction of classical decision making rather than just of probability theory and so captures the kind of reasoning possible in QPNs. 


\section{The logical language}

This section introduces the language used by our system. We build on the language of $\mathcal{Q P} \mathcal{R}$ by introducing notions of utility, but to save space here we only deal with non-categorical changes in value, simplify the language by not dealing with logical conjunction, restrict ourselves to causally directed reasoning, and cut the discussion of those features drawn from $\mathcal{Q P \mathcal { R }}$. A fuller account is contained in [19].

\subsection{Basic concepts}

We start with a set of atomic propositions $\mathcal{L}$ which includes the symbol $V$. We also have a set of connectives $\{\neg, \rightarrow, \uplus, \stackrel{v}{\rightarrow}, \sim, \stackrel{v}{\rightarrow}\}$, and the following set of rules for building the well-formed formulae ( $w f f \mathrm{~s})$ of the language.

1. If $l \in \mathcal{L}$ then $l$ is a well-formed simple formula ( $s w f f)$.

2. If $l$ is an swff, then $\neg l$ is an swff.

3. If $l$ and $m$ are $\operatorname{swffs} \mathrm{s}$, then $l \rightarrow m$ is a well-formed implicational formula (iwff).

4. If $l$ is an $s w f f$, then $l \stackrel{v}{\rightarrow} V$ is a well-formed value formula (vwff).

5. If $l, m$ and $n$ are $s w f f \mathrm{~s}$, then $l \uplus m \leadsto n$ and $l \uplus m \stackrel{v}{\sim} V$ are well-formed synergistic formulae (ywffs).

We denote the set of all swffs which can be defined using $\mathcal{L}$ by $\mathcal{S}_{\mathcal{L}}$, while $\mathcal{I}_{\mathcal{L}}$, $\mathcal{Y}_{\mathcal{L}}^{+}$and $\mathcal{V}_{\mathcal{L}}$ denote the corresponding sets of $i w f f \mathrm{~s}, y w f f \mathrm{~s}$ and $v w f f \mathrm{~s}$ respectively. The set of all wffs which can be defined using $\mathcal{L}$ is $\mathcal{W}=\mathcal{S}_{\mathcal{L}} \cup \mathcal{I}_{\mathcal{L}} \cup \mathcal{Y}_{\mathcal{L}}^{+} \cup \mathcal{V}_{\mathcal{L}}$. $\mathcal{W}$ may then be used to build up a database $\Delta$ where every item $d \in \Delta$ is a triple $(i: l: s)$ in which $i$ is a token uniquely identifying the database item (for convenience we will use the letter ' $i$ ' as an anonymous identifier), $l \in \mathcal{W}$, and $s$ gives information about the probability of $l$. In particular we take triples $(i: l: \uparrow)$ to denote the fact that $\operatorname{Pr}(l)$ increases (due to some piece of evidence), and similar triples $(i: l: \downarrow)$, to denote the fact that $\operatorname{Pr}(l)$ decreases. Triples $(i: l: \leftrightarrow)$ denote the fact that $\operatorname{Pr}(l)$ is known to neither increase nor decrease, and triples $(i: l: \uparrow)$ denote we don't know whether $\operatorname{Pr}(l)$ increases or decreases. It should be noted that the triple $(i: l: \uparrow)$ indicates that $\operatorname{Pr}(l)$ either goes up, or does not change - this inclusive interpretation of the notion of "increase" is taken from QPNs — and of course a similar proviso applies to $(i: l: \downarrow)$.

\subsection{Non-material implication}

Now, " $\rightarrow$ " does not represent material implication but a connection between the probabilities of antecedent and consequent. We take an iwff, which we will also call an "implication", to denote that the antecedent of the iwff has a probabilistic influence on the consequent. Thus we are not concerned with the probability of the iwff, but what the wff says about the probabilities of its antecedent and 
consequent. More precisely we take the triple $(i: a \rightarrow c:+)$ to denote the fact that:

$$
\operatorname{Pr}(c \mid a, X) \geq \operatorname{Pr}(c \mid \neg a, X)
$$

for all $X \in\{x, \neg x\}$ for which there is a triple $(i: X \rightarrow c: s)$ (where $s$ is any sign) or $(i: c \rightarrow X: s)$. The effect of the $X$ in this inequality is to ensure that the restriction holds whatever is known about formulae other than $c$ and $a$-whatever the probabilities of $a$ and $c$, the constraint on the conditional probabilities holds. It is possible to think of this as meaning that there is a constraint on the probability distribution over the formulae $c$ and $a$ such that an increase in the probability of $a$ entails an increase in the probability of $c$. The triples $(i: a \rightarrow c:-)$ and $(i: a \rightarrow c: 0)$ denote that (1) holds with $\geq$ replaced by $\leq$ and $=$ respectively. We also have implications such as $(i: a \rightarrow c: ?)$ which denotes the fact that the relationship between $\operatorname{Pr}(c \mid a, X)$ and $\operatorname{Pr}(c \mid \neg a, X)$ is not known, so that if the probability of $a$ increases it is not possible to say how the probability of $c$ will change.

With this interpretation, implications correspond to qualitative influences in QPNs, and, as is the case in all probabilistic networks, [20] are causally directed in the sense that the antecedent is a cause of the consequent. This restriction is necessary to ensure that $\mathcal{Q U \mathcal { R }}$ is sound, for the reasons discussed in [17].

\section{$2.3 \quad$ Values}

The proposition $V$ denotes the same thing as the value node in an influence diagram [13] - that is the utility of the decision maker. It can be used, just like any other swff to form triples, and these denote a change in utility. Thus $(i: V: \uparrow)$ means that utility increases. $\mathcal{Q U} \mathcal{R}$ also makes use of triples based on $v w f f \mathrm{~s}$, and a $v w f f(i: a \stackrel{v}{\rightarrow} V:+)$ is taken to mean:

$$
U(a, X) \geq U(\neg a, X)
$$

where, as before, $X$ ranges across all other propositions which affect $V$, in this case all other propositions which are antecedents of vwffs. The meaning of the triple, as given by (2), is that $a$ positively influences utility. Similar triples with sign - and 0 denote that $(2)$ holds with $\geq$ replaced by $\leq$ and $=$ respectively, and we use the sign ? to denote situations in which the relationship is not known.

\subsection{Synergy}

Being able to handle synergy relations is an important part of any qualitative probabilistic system. A detailed discussion of synergy is beyond the scope of this paper ${ }^{1}$, but, informally, there is synergy between two variables with respect to a third if a change in the value of one of the first two has an effect on the relationship between the second and the third. Thus, $A$ and $B$ have a synergistic relationship with respect to $C$, if an increase in the probability of $A$ changes the

\footnotetext{
${ }^{1}$ See $[5,6,25]$ for detail on the subject.
} 
strength of the probabilistic influence between $B$ and $C$. In our system synergies are represented by formulae such as $a \uplus b \leadsto c$ which represents the synergy which exists between $a$ and $b$ with respect to $c$. Such synergistic formulae form the basis of triples such as $(i: a \uplus b \leadsto c:+)$ in just the same way as simple and implicational formulae do, but with yet another denotation. In particular, $(i: a \uplus b \leadsto c:+)$ denotes the fact that:

$$
\operatorname{Pr}(c \mid a, b, X)+\operatorname{Pr}(c \mid \neg a, \neg b, X) \geq \operatorname{Pr}(c \mid \neg a, b, X)+\operatorname{Pr}(c \mid a, \neg b, X)
$$

where as ever, $X$ ranges across all other formulae such that there are triples $(i: X \rightarrow c: s)$ or $(i: c \rightarrow X: s)$. Similarly, $(i: a \uplus b \leadsto c:-)$ and $(i: a \uplus b \leadsto c: 0)$ denote that $(3)$ holds with $\geq$ replaced by $\leq$ and $=$ respectively. As with the case of implications, synergies have sign ? when the relationship is not known. These synergy expressions are $[18,25]$ precisely the conditions necessary and sufficient to capture the fact that a change in $\operatorname{Pr}(a)$ has an effect on the influence of $\operatorname{Pr}(b)$ on $\operatorname{Pr}(c)$. It is perfectly possible to have synergies with respect to the value node represented by triples such as $(i: a \uplus b \stackrel{v}{\sim} V:+)$. This latter denotes the fact that:

$$
U(a, b, X)+U(\neg a, \neg b, X) \geq U(\neg a, b, X)+U(a, \neg b, X)
$$

where $X$ is as before. Similarly, $(i: a \uplus b \leadsto V:-)$ and $(i: a \uplus b \leadsto V: 0)$ denote that $(4)$ holds with $\geq$ replaced by $\leq$ and $=$ respectively. Note that all synergies are symmetrical, and that the synergies we deal with here are known as additive synergies. In contrast, $\mathcal{Q P} \mathcal{R}[17]$ deals only with product synergies.

\section{The proof theory}

The previous section introduced a language for describing probabilistic influences between formulae. For this to be useful, we need to give a mechanism for taking sentences in that language and using them to derive new sentences.

\subsection{Arguments}

We derive new sentences using the consequence relation $\vdash_{Q U}$ which is defined in Figure 1. The definition is in terms of Gentzen-style proof rules where the antecedents are written above the line and the consequence is written below. The consequence relation operates on a database consisting of the kind of triples introduced in the previous section and derives arguments about formulae from them. There are two types of argument $^{2}$ :

Definition 1. An influence argument for a well-formed formula p from a database $\Delta$ is a triple $S(p, G, s)$ such that $\Delta \vdash_{Q U} S(p, G, s)$

The sign $s$ of an influence argument denotes something about the change in the probability of $p$ which can be inferred given the grounds $G$-the elements of the database used in the derivation of $p$.

\footnotetext{
${ }^{2}$ The use of $S$ and $Y$ to denote the different types is taken from [25].
} 


$$
\begin{aligned}
& \text { S-rules } \\
& \operatorname{Ax} 1 \frac{}{\Delta \vdash_{Q U} S(S t,\{i\}, S g)}(i: S t: S g) \in \Delta, S t \in \mathcal{S}_{\mathcal{L}} \cup \mathcal{I}_{\mathcal{L}} \cup \mathcal{V}_{\mathcal{L}} \\
& \neg-\mathrm{E} \frac{\Delta \vdash_{Q U} S(\neg S t, G, S g)}{\Delta \vdash_{Q U} S(S t, G, \operatorname{neg}(S g))} \\
& \neg-\mathrm{I} \frac{\Delta \vdash_{Q U} S(S t, G, S g)}{\Delta \vdash_{Q U} S(\neg S t, G, \operatorname{neg}(S g))} \\
& \rightarrow-\mathrm{E} \frac{\Delta \vdash_{Q U} S(S t, G, S g) \Delta \vdash_{Q U} S\left(S t \rightarrow S t^{\prime}, G^{\prime}, S g^{\prime}\right)}{\Delta \vdash_{Q U} S\left(S t^{\prime}, G \cup G^{\prime}, \operatorname{impelim}_{\text {elim }}\left(S g, S g^{\prime}\right)\right)}
\end{aligned}
$$

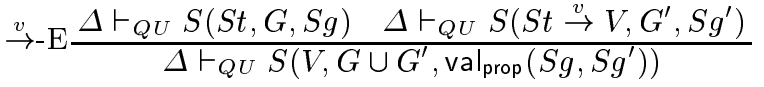

$$
\begin{aligned}
& \mathrm{Ax} 2 \frac{}{\Delta \vdash_{Q U} Y\left(\left(S t^{\prime \prime}, S t, S t^{\prime}\right),\{i\}, S g\right)}\left(i: S t \uplus S t^{\prime} \leadsto S t^{\prime \prime}: S g\right) \in \Delta \\
& \mathrm{Ax} 3 \frac{}{\Delta \vdash_{Q U} Y\left(\left(V, S t, S t^{\prime}\right),\{i\}, S g\right)}\left(i: S t \uplus S t^{\prime} \stackrel{v}{\sim} V: S g\right) \in \Delta \\
& \mathrm{Y}-\mathrm{I} 1 \frac{\Delta \vdash_{Q U} S\left(S t \rightarrow S t^{\prime}, G, S g\right) \Delta \vdash_{Q U} Y\left(\left(S t, S t^{\prime \prime}, S t^{\prime \prime \prime}\right), G^{\prime}, S g^{\prime}\right)}{\Delta \vdash_{Q U} Y\left(\left(S t^{\prime}, S t^{\prime \prime}, S t^{\prime \prime \prime}\right), G \cup G^{\prime}, \operatorname{syn}_{\text {prop }}\left(S g, S g^{\prime}\right)\right)} \\
& \mathrm{Y}-\mathrm{I} 2 \frac{\Delta \vdash_{Q U} S\left(S t \rightarrow S t^{\prime}, G, S g\right) \quad \Delta \vdash_{Q U} Y\left(\left(S t^{\prime \prime}, S t^{\prime}, S t^{\prime \prime \prime}\right), G^{\prime}, S g^{\prime}\right)}{\Delta \vdash_{Q U} Y\left(\left(S t^{\prime \prime}, S t, S t^{\prime \prime \prime}\right), G \cup G^{\prime}, \operatorname{syn}_{\text {prop }}\left(S g, S g^{\prime}\right)\right)} \\
& \mathrm{Y}-\mathrm{I} 3 \frac{\Delta \vdash_{Q U} S\left(S t \rightarrow S t^{\prime}, G, S g\right) \quad \Delta \vdash_{Q U} Y\left(\left(S t^{\prime \prime}, S t^{\prime \prime \prime}, S t^{\prime}\right), G^{\prime}, S g^{\prime}\right)}{\Delta \vdash_{Q U} Y\left(\left(S t^{\prime \prime}, S t, S t^{\prime \prime \prime}\right), G \cup G^{\prime}, \operatorname{syn}_{\text {prop }}\left(S g, S g^{\prime}\right)\right)}
\end{aligned}
$$

Fig. 1. The consequence relation $\vdash_{Q U}$

Definition 2. A synergy argument for a well-formed formula $p$ from a database $\Delta$ is a triple $Y((p, q, r), G, s)$ such that $\Delta \vdash_{Q U} Y((p, q, r), G, s)$

Such an argument indicates that $q$ and $r$ have a synergistic effect on $p$. The sign gives the synergy of $q$ on the relation between $r$ and $p$, or, equivalently, the synergy of $r$ on the relation between $q$ and $p$.

To see how the idea of an argument fits in with the proof rules in Figure 1, consider the rules 'Ax1', and ' $\rightarrow$-E'. The first says that from a triple $(i: l: s)$ it is possible to build an argument for $l$ which has sign $s$ and a set of grounds $\{i\}$ (the grounds thus identify which elements from the database are used in the derivation). The rule is thus a kind of bootstrap mechanism to allow the elements of the database to be turned into arguments to which other rules can 


\begin{tabular}{|c|cccc|}
\hline$s$ & $\uparrow$ & $\leftrightarrow$ & $\downarrow$ & $\downarrow$ \\
\hline $\operatorname{neg}(s)$ & $\downarrow$ & $\leftrightarrow$ & $\uparrow$ & $\downarrow$ \\
\hline
\end{tabular}

\begin{tabular}{|c|cccc|}
\hline impelim $_{\text {el }}$ & + & 0 & - & $?$ \\
\hline$\uparrow$ & $\uparrow$ & $\leftrightarrow$ & $\downarrow$ & $\uparrow$ \\
$\leftrightarrow$ & $\leftrightarrow$ & $\leftrightarrow$ & $\leftrightarrow$ & $\leftrightarrow$ \\
$\downarrow$ & $\downarrow$ & $\leftrightarrow$ & $\uparrow$ & $\uparrow$ \\
$\uparrow$ & $\uparrow$ & $\leftrightarrow$ & $\uparrow$ & $\uparrow$ \\
\hline
\end{tabular}

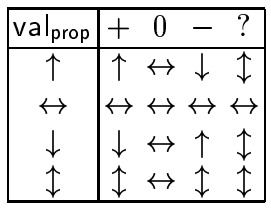

Fig. 2. The functions neg, impelim and val $\mathrm{p}_{\text {prop }}$.

be applied. The second rule ' $\rightarrow$-E' can be thought of as analogous to modus ponens. From an argument for $a$ and an argument for $a \rightarrow c$ it is possible to build an argument for $c$ once the necessary book-keeping with grounds and signs has been carried out. The proof procedure used here has an important difference from other similar logical proof systems which stems from the fact that $\mathcal{Q U} \mathcal{R}$ is dealing with probability values (albeit changes in probability) rather than just truth and falsity as is the case in classical logic. In logic, once there is a valid proof for a formula, the formula is known to be true. Here we may have several arguments which suggest different things about the probability of a formula and it is necessary to establish all the arguments and then combine them.

\subsection{Combination functions}

In order to apply the proof rules to build arguments, it is necessary to supply the functions used in Figure 1 to combine signs. Broadly speaking, all these functions are exactly those introduced by Wellman [25] for the analogous operations in $\mathrm{QPNs}^{3}$. The rules for handling negation are applicable only to swffs and permit negation to be either introduced or eliminated by altering the sign, for example allowing $(i: \neg a: \uparrow)$ to be rewritten as $(i: a: \downarrow)$. This leads to the definition of neg:

Definition 3. The function neg : $S g \in\{\uparrow, \leftrightarrow, \downarrow, \uparrow\} \mapsto S g^{\prime} \in\{\uparrow, \leftrightarrow, \downarrow, \uparrow\}$ is specified in Figure 2.

To deal with implication we need the function impelim to establish the sign of formulae generated by the rule of inference $\rightarrow$-E. This means that impelim is used to combine the change in probability of a formula $a$, say, with the constraint that the probability of $a$ imposes upon the probability of another formula $c$.

Definition 4. The function impelim $_{\text {e }} S g \in\{\uparrow, \leftrightarrow, \downarrow, \uparrow\} \times S g^{\prime} \in\{+, 0,-, ?\} \mapsto$ $S g^{\prime \prime} \in\{\uparrow, \leftrightarrow, \downarrow, \uparrow\}$ is specified in Figure 2.

We also need the function val $\left.\right|_{\text {prop }}$ which makes it possible to determine the changes in utility.

Definition 5. The function val prop $: S g \in\{\uparrow, \leftrightarrow, \downarrow, \uparrow\} \times S g^{\prime} \in\{+, 0,-, ?\} \mapsto$ $S g^{\prime \prime} \in\{\uparrow, \leftrightarrow, \downarrow, \uparrow\}$ is specified in Figure 2.

${ }^{3}$ The reason our notation differs is to allow our system to be extended to handle categorical information exactly as in [17]. 


\begin{tabular}{|c|c|}
\hline syn $_{\text {prop }}$ & $+0-?$ \\
\hline+ & $+0-?$ \\
\hline 0 & $\mid \begin{array}{llll}0 & 0 & 0 & ?\end{array}$ \\
\hline- & $-0+?$ \\
\hline ? & ? ? ? ? \\
\hline
\end{tabular}

\begin{tabular}{|c|cccc|}
\hline flats & $\uparrow$ & $\leftrightarrow$ & $\downarrow$ & $\uparrow$ \\
\hline$\uparrow$ & $\uparrow$ & $\uparrow$ & $\uparrow$ & $\uparrow$ \\
$\leftrightarrow$ & $\uparrow$ & $\leftrightarrow$ & $\downarrow$ & $\uparrow$ \\
$\downarrow$ & $\uparrow$ & $\downarrow$ & $\downarrow$ & $\uparrow$ \\
$\uparrow$ & $\uparrow$ & $\uparrow$ & $\uparrow$ & $\uparrow$ \\
\hline
\end{tabular}

\begin{tabular}{|c|cccc|}
\hline flat & + & 0 & - & $?$ \\
\hline+ & + & + & $?$ & $?$ \\
0 & + & 0 & - & $?$ \\
- & $?$ & - & - & $?$ \\
$?$ & $?$ & $?$ & $?$ & $?$ \\
\hline
\end{tabular}

Fig. 3. Synergy propagation syn $n_{\text {prop }}$ and flattening functions flats and flaty.

This function is virtually identical to impelim, differing only in that it combines a change in probability with a utility to give a change in expected utility, whereas impelim derives a change in probability from a change in probability and a relationship between probabilities. We also need the function $\operatorname{syn}_{\text {prop }}$ in order to be able to reason with synergies.

Definition 6. The function syn $_{\text {prop }}: S g \in\{+, 0,-, ?\} \times S g^{\prime} \in\{+, 0,-, ?\} \mapsto$ $S g^{\prime \prime} \in\{+, 0,-, ?\}$ is specified in Figure 3 .

These functions are sufficient to apply $\vdash_{Q U}$ to build both influence and synergy arguments.

\subsection{Flattening}

In general it is possible to build several arguments for a single proposition. To get firm conclusions we need to flatten all the arguments for a proposition to get a single sign which tells us the combined change in the probability of that proposition. We can describe this in terms of a function Flat $_{S}(\cdot)$ which maps from a set of influence arguments $\mathbf{A}_{\mathbf{S}}$ for a proposition $S t$ built from a particular database $\Delta$ to the pair of that proposition and some overall measure of validity:

$$
\text { Flat }_{\mathrm{S}}: \mathbf{A}_{\mathbf{S}} \mapsto S\langle S t, v\rangle
$$

where $\mathbf{A}_{\mathbf{S}}$ is the set of all influence arguments which are concerned with $S t$, that is:

$$
\mathbf{A}_{\mathbf{S}}=\left\{S\left(S t, G_{i}, S g_{i}\right) \mid \Delta \vdash_{Q U} S\left(S t, G_{i}, S g_{i}\right)\right\}
$$

and $v$ is the result of a suitable combination of the $S g$ that takes into account the structure of the arguments. Since in the precise case we are considering here, the structure is unimportant (though in very similar cases it must be taken into consideration [17]) we can ignore the grounds and define $v$ as:

$$
v=\operatorname{flat}_{\mathbf{S}}\left(\left\{S g_{i} \mid\left(S t, G_{i}, S g_{i}\right) \in \mathbf{A}_{\mathbf{S}}\right\}\right)
$$

where flats is as defined in Figure 3. We can formalise a similar notion for synergy arguments in terms of a function Flat $(\cdot)$ which maps from a set of synergy arguments $\mathbf{A}_{\mathbf{Y}}$ for a proposition $S t$ to the pair of that synergistic relationship and some overall measure of validity:

$$
\text { Flat } \mathbf{A}_{\mathbf{Y}} \mapsto Y\left\langle\left(S t, S t^{\prime}, S t^{\prime \prime}\right), v\right\rangle
$$


where $\mathbf{A}_{\mathbf{Y}}$ is the set of all synergy arguments which give the synergistic effect of $S t^{\prime}$ and $S t^{\prime \prime}$ on $S t$ :

$$
\begin{aligned}
\mathbf{A}_{\mathbf{Y}}=\left\{Y\left(\left(S t, S t^{\prime}, S t^{\prime \prime}\right), G_{i}, S g_{i}\right) \mid\right. & \Delta \vdash_{Q U} Y\left(\left(S t, S t^{\prime}, S t^{\prime \prime}\right), G_{i}, S g_{i}\right) \\
& \text { or } \left.\Delta \vdash_{Q U} Y\left(\left(S t, S t^{\prime \prime}, S t^{\prime}\right), G_{i}, S g_{i}\right)\right\}
\end{aligned}
$$

and $v$ is defined by:

$$
v=\operatorname{flat}_{\mathbf{Y}}\left(\left\{S g_{i} \mid\left(\left(S t, S t^{\prime}, S t^{\prime \prime}\right), G_{i}, S g_{i}\right) \in \mathbf{A}_{\mathbf{Y}}\right\}\right)
$$

where flat $t_{Y}$ is given in Figure 3.

\section{Soundness and Completeness}

We can show that $\mathcal{Q U} \mathcal{R}$ is sound with respect to decision theory, and determine bounds on what it can deduce. First consider soundness ${ }^{4}$ :

Theorem 1. The construction and flattening of influence and synergy arguments in $\mathcal{Q U R}$ using $\vdash_{Q U}$ is sound with respect to decision theory.

To prove completeness, one first needs to establish a proof procedure. The procedure for computing the effect on some formula $p$ is:

1. Add a triple $(i: q: s)$ for every formula $q$ whose change in probability is known.

2. Build $\mathbf{A}_{\mathbf{S}}$, the set of all influence arguments for $p$.

3. Flatten this set to $S\left\langle p, v_{S}\right\rangle$.

4. Build $\mathbf{A}_{\mathbf{Y}}$, the set of all synergy arguments for $p$.

5. Flatten this set to $Y\left\langle p, v_{Y}\right\rangle$.

This naturally backward chaining procedure can obviously be extended to compute the effect on a whole set of propositions. Now, we also need to define the sense in which we consider the system to be complete.

Definition 7. A well-formed formula $p$ is said to be a cause of a well-formed formula $q$ if and only if it is possible to identify an ordered set of iwffs $\{p \rightarrow$ $\left.a_{1}, a_{1} \rightarrow a_{2}, \ldots, a_{n} \rightarrow q\right\}$. If $q$ is the value proposition $V$, the final member of the set is $a_{n} \stackrel{v}{\rightarrow} V$.

In other words $p$ is a cause of $q$ if it is possible to build up a trail of (causally directed) implications which link $p$ to $q$. We have a similar notion for synergies:

Definition 8. A well-formed formula $p$ is said to be a synergistic cause of a well-formed formula $q$ if there is a ywff $a \uplus b \leadsto c$ such that $p$ is a cause of either $a$ or $b$ and $c$ is a cause of $q$. If $q$ is the value proposition $V$, then the ywff in question is of the form $a \uplus b \stackrel{v}{\sim} V$.

\footnotetext{
${ }^{4}$ All the proofs in this section are straightforward but lengthy, and so have been omitted to save space. They may be found in [19] and are simple extensions of those in [17].
} 
Definition 9. A well-formed formula $q$ is said to be an effect (respectively a synergistic effect) of a well-formed formula $p$ if and only if $p$ is a cause (respectively a synergistic cause) of $q$.

Definition 10. The construction and flattening of arguments is said to be causally complete in some system of qualitative utility with respect to some formula $p$ if it is possible to use that system to compute the changes in probability of all the effects of $p$.

Given these definitions we can prove that $\mathcal{Q U} \mathcal{R}$ is complete in the following sense:

Theorem 2. The construction and flattening of influence arguments in $Q \mathcal{U R}$ using $\vdash_{Q U}$ is causally complete with respect to any simple well-formed formula.

We also need to deal with synergy arguments. For them we need the following notion of completeness:

Definition 11. The construction and flattening of arguments is said to be synergistically causally complete in some system of qualitative utility with respect to some formula $p$ if it is possible to use that system to compute the synergies involving $p$ and all its synergistic effects.

Given this we can show that:

Theorem 3. The construction and flattening of synergy arguments in $\mathcal{Q U R}$ using $\vdash_{Q U}$ is synergistically causally complete with respect to any simple wellformed formula.

Note that completeness is defined only in terms of swffs. This restriction is considered in detail in [19].

\section{Example}

This section presents a short example of the kind of reasoning possible in $\mathcal{Q U} \mathcal{R}$. Since the example is one used in [25], it also helps to informally demonstrate the fact that $\mathcal{Q U R}$ captures the kind of reasoning possible in QPNs.

The example concerns the decisions made about digitalis therapy, and comes initially from [22]. An increased dosage of digitalis (dig) has a negative effect on conduction (con) ( $r 1)$ and a positive effect on automaticity (aut) (r2). A negative effect on conduction is the aim of the therapy since the conduction has a positive effect on heart rate $(h r)(r 3)$ and a reduction in heart rate is what is required $(r 4)$. Automaticity has a positive effect on ventricular fibrillation $(v f)$ $(r 5)$, a life threatening state $(r 6)$. High calcium levels $(\mathrm{Ca})$ also have a positive effect on automaticity $(r 7)$. Increasing the digitalis dose makes automaticity more sensitive to calcium level $(r 8)$, and an increased heart rate means that 
ventricular fibrillation has a more severe effect on the patient's well-being. This information can be expressed as:

$$
\begin{aligned}
& (r 1: \operatorname{dig} \rightarrow \text { con }:-) \quad(r 4: h r \stackrel{v}{\rightarrow} V:-) \quad(r 7: C a \rightarrow \text { aut }:+) \quad \Delta_{1} \\
& (r 2: \operatorname{dig} \rightarrow \text { aut }:+) \quad(r 5: \text { aut } \rightarrow \text { vf }:+) \quad(r 8: \operatorname{dig} \uplus C a \leadsto a u t:+) \\
& (r 3: \text { con } \rightarrow h r:+) \quad(r 6: v f \stackrel{v}{\rightarrow} V:-) \quad(r 9: h r \uplus v f \stackrel{v}{\sim} V:+)
\end{aligned}
$$

Adding $(f 1: \operatorname{dig}, \uparrow)$, indicating increased digitalis dosage, to this database, we can build the influence arguments:

$$
\begin{aligned}
& S(V,\{r 1, r 3, r 4\}, \uparrow) \\
& S(V,\{r 2, r 5, r 6\}, \downarrow)
\end{aligned}
$$

These indicate, respectively, that there are reasons to both think that overall utility will increase and that it will decrease. These flatten to give $S\langle V, \uparrow\rangle$ indicating, exactly as with the equivalent QPN, that there is no conclusive argument. We can also build two synergy arguments connecting $\operatorname{dig}$ and $C a$ with $V$ :

$$
\begin{aligned}
& Y((V, C a, d i g),\{r 8, r 5, r 6\},-) \\
& Y((V, \operatorname{dig}, C a),\{r 9, r 5, r 7, r 3, r 1\},-)
\end{aligned}
$$

These flatten to give $Y\langle(V$, dig, $C a),-\rangle$, indicating that digitalis dosage and calcium level have a negative synergistic effect on overall utility. Thus increasing digitalis dosage reduces the effect that an increase in calcium level has on utility.

\section{Discussion}

The system introduced in this paper has its roots in Wellman's QPNs [25], the first attempt to build a qualitative decision theory, and draws its notion of "qualitative" from QPNs. This is a notion close to that in qualitative physics [14] where the basic abstraction is that which distinguishes between positive, negative and zero quantities and the derivatives of those quantities. The main focus in both QPNs and $\mathcal{Q U} \mathcal{R}$ is on the way in which values change with evidence. These two factors, the extreme abstraction and the concentration on change, distinguishes both $\mathcal{Q U} \mathcal{R}$ and QPNs from other qualitative systems.

As mentioned in the introduction, there have been a number of attempts to devise qualitative decision theories where "qualitative" is taken to means some form of relative order of magnitude based upon infinitesimal quantities. The first such effort was that of Pearl [21] which abstracted utility values in this way (earlier work, such as that of Darwiche [3] and Goldszmidt [12] had dealt with probabilities of this form). In doing this, Pearl thus provided an order of magnitude version of classical decision theory. This was then extended by Tan $[23,24]$ to deal with conditional preferences, so that it is possible to base decisions on statements like "if $\beta$ is preferred to $\alpha$ ". Around the same time Wilson [26] provided an alternative way to formulate Pearl's original qualitative version of classical decision theory, and more recently Lehmann [15] has made a similar 
proposal. The strand of this work which is most similar to ours is that of Bonet and Geffner [2], who also keep track of the reasons behind the decision, in terms of the information used to reach it.

The use of a different notion of "qualitative" is that investigated by Dubois, Prade and colleagues [7-9]. Their system has a possibilistic rather than a probabilistic semantics and is qualitative in the sense that only the ordinal rank of quantities is important. It should be noted, however, that the values they use are not infinitesimal (though one could build an infinitesimal version of their theory), and so can be considered more expressive than those of Pearl et al. It should also be noted that while, as described here, our system has a probabilistic semantics, we can give it alternative semantics, as discussed in [19].

\section{Summary}

This paper has extended our previous work on proof theoretic approaches to qualitative probabilistic reasoning [17] in two important ways. First this paper has extended it to deal with statements of utility, making it possible to reason about changes in expected utility as well as about changes in probabilities. This is an important step in developing a qualitative decision theory. Second, this paper has dealt with the concept of additive synergy, which is important in determining dominating decision options [25].

Acknowledgments This work was partly funded by the EPSRC under grant GR/L84117. The authors are grateful to Peter McBurney and the anonymous referees for their comments on an earlier version of this paper.

\section{References}

1. S. Benferhat, D. Dubois, and H. Prade. Argumentative inference in uncertain and inconsistent knowledge bases. In Proceedings of the 9th Conference on Uncertainty in Artificial Intelligence, 1993.

2. B. Bonet and H. Geffner. Arguing for decisions: a qualitative model of decision making. In Proceedings of the 12th Conference on Uncertainty in Artificial Intelligence, 1996.

3. A. Darwiche. A symbolic generalization of probability theory. PhD thesis, Stanford University, 1993.

4. A. Darwiche and M. Goldszmidt. On the relation between kappa calculus and probabilistic reasoning. In Proceedings of the 10th Conference on Uncertainty in Artificial Intelligence, 1994.

5. M. J. Druzdzel. Probabilistic reasoning in decision support systems: from computation to common sense. PhD thesis, Carnegie Mellon University, 1993.

6. M. J. Druzdzel and M. Henrion. Intercausal reasoning with uninstantiated ancestor nodes. In Proceedings of the 9th Conference on Uncertainty in Artificial Intelligence, 1993. 
7. D. Dubois, L. Godo, H. Prade, and A. Zapico. Making decision in a qualitative setting: from decision under uncertainty to case-based decision. In Proceedings of the 6th International Conference on Knowledge Representation and Reasoning, 1998.

8. D. Dubois and H. Prade. Possibility theory as a basis for qualitative decision theory. In Proceedings of the 14th International Joint Conference on Artificial Intelligence, 1995.

9. D. Dubois, H. Prade, and R. Sabbadin. A possibilistic logic machinery for qualitative decision. In Proceedings of the AAAI Spring Symposium on Qualitative Preferences in Deliberation and Practical Reasoning, 1997.

10. P. M. Dung. On the acceptability of arguments and its fundamental role in nonmonotonic reasoning and logic programming. In Proceedings of the 13th International Joint Conference on Artificial Intelligence, 1993.

11. J. Fox. A unified framework for hypothetical and practical reasoning (2): lessons from clinical medicine. In Proceedings of the Conference on Formal and Applied Practical Reasoning, 1996.

12. M. Goldszmidt. Qualitative probabilities: a normative framework for commonsense reasoning. PhD thesis, University of California at Los Angeles, 1992.

13. R. A. Howard and J. E. Matheson. Influence diagrams. In R. A. Howard and J. E. Matheson, editors, Readings on the Principles and Applications of Decision Analysis, pages 719-762. Strategic Decisions Group, Menlo Park, CA, 1984.

14. B. Kuipers, editor. Qualitative Reasoning-Modelling and Simulation with Incomplete Knowledge. MIT Press, Cambridge, MA, 1994.

15. D. Lehmann. What is qualitative? a framework for quantitative and qualitative decision theory. In Proceedings of the AAAI Spring Symposium on Qualitative Preferences in Deliberation and Practical Reasoning, 1997.

16. R. Loui. Defeat among arguments: a system of defeasible inference. Computational Intelligence, 3:100-106, 1987.

17. S. Parsons. A proof theoretic approach to qualitative probabilistic reasoning. International Journal of Approximate Reasoning, 19:265-297, 1998.

18. S. Parsons. Qualitative approaches to reasoning under uncertainty. MIT Press, (to appear), Cambridge, MA, 1999.

19. S. Parsons and S. Green. A proof theoretic approach to qualitative decision making. Technical report, Department of Electronic Engineering, Queen Mary and Westfield College, 1999.

20. J. Pearl. Probabilistic reasoning in intelligent systems; networks of plausible inference. Morgan Kaufmann, San Mateo, CA., 1988.

21. J. Pearl. From conditional oughts to qualitative decision theory. In Proceedings of the 9th Conference on Uncertainty in Artificial Intelligence, 1993.

22. W. Swartout. XPlain: a system for creating and explaining expert consulting programs. Artificial Intelligence, 21:285-325, 1983.

23. Sek-Wah Tan and J. Pearl. Qualitative decision theory. In Proceedings of the 12th National Conference on Artificial Intelligence, 1994.

24. Sek-Wah Tan and J. Pearl. Specification and evaluation of preferences under uncertainty. In Proceedings of the 4th International Conference on Knowledge Representation and Reasoning, 1994.

25. M. P. Wellman. Formulation of tradeoffs in planning under uncertainty. Pitman, London, 1990.

26. N. Wilson. An order of magnitude calculus. In Proceedings of the 11th Conference on Uncertainty in Artificial Intelligence, 1995. 